\section{Scherer, Johann Joseph von}

\author{
A. M. Gressner ${ }^{1}$ und O. A. Gressner ${ }^{2}$ \\ ${ }^{1}$ Labor Dr. Wisplinghoff Berlin, Berlin, Deutschland \\ ${ }^{2}$ Labor Dr. Wisplinghoff Köln, Köln, Deutschland
}

Lebensdaten Deutscher Chemiker und Mediziner, geboren am 13. März 1814 in Aschaffenburg, gestorben am 17. Februar 1869 in Würzburg.

Verdienste Scherer studierte in Würzburg Medizin und in München Chemie und war Assistent bei Justus von Liebig in Gießen. Ab 1847 ordentlicher Professor für Organische Chemie und Leiter des klinisch-chemischen Laboratoriums am
Julius-Hospital in Würzburg. Klinisch-chemische und pathologisch-chemische Arbeiten zur Analyse des Bluts in enger Verbindung mit der Klinik führten u. a. zur Entdeckung von $>$ Hypoxanthin und (Meso-)Inosit. Scherer gilt mit Johann Florian Heller ( $\vee$ Heller, Johann Florian) und Johann Franz Simon ( $\triangleright$ Simon, Johann Franz) als Begründer der Klinischen Chemie ( $\triangleright$ Klinische Chemie) in Deutschland.

\section{Literatur}

Grund CR (2002) Der Würzburger Chemiker Johann Joseph von Scherer und die Begründung der Klinischen Chemie im 19. Jahrhundert. Shaker Verlag, Aachen 